simple or invading or destructive may arise from some bodily defect; a youth with a squint thinks that he is shunned. He has found that at school he was called "cock eye." This has, acting on a sensitive fellow, led to isolation and general exaggerated self-consciousness till at last, though admitted to be a man of ability, he secludes himself and studies but cannot face the world. A deaf man in similar circum. stances thinks that people are talking about him and acts similarly. Such cases, as I say, may pass beyond the simple stage, and their conduct may require their supervision. The emotional feelings may in a similar way lead to mental disorder. The anxious wife may become the suspicious, jealous, and, finally, the insanely jealous one. In some such cases the growth is simple but in others it is spreading.

Next, as to some of the more marked invading growths in which there is little or no mental decay. The very best are seen in some cases of hypochondriasis : a man believes that he has some throat affection, he goes from specialist to specialist, from country to country, nothing is able to satisfy him, his whole life and interest is bound up in his ailment, he ceases to take any interest in anything, he would not be able to earn a living, and drifts into a home or an asylum. In some such cases a dominating cause can be traced. In one gentleman the fact that he had been bitten by a dog led him to go to Paris to undergo Pasteur treatment. He has never been able to throw off the dread of rabies. In one case, that of a talented doctor who belonged to a highly distinguished but unstable family, the idea of brain disease has so controlled his life that he has been confined in asylums and will be to the end. He can do nothing for himself but his mind is clear, his memory is remarkably good, and he is still learning and advancing in general knowledge.

There is a difficulty in separating the cases in which simple invasion takes place from those in which there is invasion with destruction, for in the earlier stages they are exactly alike. The cases of organised delusional insanity provide the best examples of invasion with destruction. In many cases it is easy to trace the origin of the delusion to some outside cause. An only son living with his mother gradually gets into solitary ways and later thinks that he is accused of criminal sexual vices in many cases he has been a secret sinner for years, and the consciousness of his fault being projected to the minds of others seems simple. The man who marries his mistress or a woman of a lower order not infrequently finds that she, knowing his failings, is persuaded that he is no longer faithful to her and hence arise all sorts of misgivings, suspicions jealousies, and fully organised delusions of suspicion. The man with a specific scar may grow to think that his fellows know the origin of the scar and first avoid him, then make remarks about it and him, and thus he may have a fully organised set of delusions of persecution and annoyance. The patient with any sexual disorder is among the most likely to develop delusions that other people know all about it. Thus a young woman with too frequent menstruation was certain that even the railway guards knew this and repeated to one another " poor little three weeks." But I have given enough examples to show what I mean when I say that there are certain disorders of mind which I cannot attribute to the removal of higher functions. They are cases in which there has never been a full growth of the power of normal social adaptation.

Now, to sum up, it is not for everyone to become sane there is no one standard of sanity and there is no one patho logy. There is, therefore, the personal factor in every case of insanity; next the dissolution of mental faculties explain many but not all the forms of insanity. The result varies with the rate of dissolution, with the depth of dissolution, and with the cause and the general conditions of the person suffer ing. The symptoms rarely depend on dissolution alone ; beside the loss of the higher faculties there may be developments of the remaining faculties leading to variations in the results.

Beside the insanities of dissolution $I$ have pointed out that there are morbid mental growths of var ous kinds, the simple, the invading, and the malignant or destructive. Much insanity depends on brain disorder but much also arises from social misfitting.

River Pollution.-At a meeting of the Somerset connty council held on Oct. 15th, owing to further complaints having been received as to the state of the bed of the River Yeo, caused by the discharge of the sewage of Yeovil, it was decided to apply to the county court to enforce the injunction already obtained against the corporation of Yeovil.

\section{ON MOBILITY OF THE KIDNEYS.}

BX HECTOR MACKENZIE, M.D. CANTAB., F.R.C.P. LOND.,

PHYSICLAN TO ST. THOMAS'S HOSPITAL AND TO THE BROMPTON HOSPITAL FOR CONSUMPTION.

TuE communication which I have the honour to bring before you to-night is the outcome of work carried on over a considerable period of time in the out-patient departments and wards of two large hospitals, St. Thomas's and Brompton. The observations of the cases which I have here recorded were mostly made several years ago when I had charge of out-patients and worked in a field which gives almost unlimited opportunity for making an investigation of this kind. The investigation includes the examination of the abdomen of nearly 4000 persons. The great majority of the patients were examined by myself personally and I verified the fact of the mobility of the kidneys in all cases, but I must gratefully acknowledge the valuable assistance afforded me by my former clinical assistants, especially Dr. Andrew Elliot, Dr. J. Douglas Cree, and Mr. R. N. Daniel. The subject of mobility of the kidneys is one of much clinical interest and importance. It is important on account of the great frequency with which abnormal mobility is to be observed among female patients when examina. tion is made in the proper way. Nephroptosis is an undoubted cause of suffering and inconvenience or actual distress and illness in many cases, and when it is recognised as their cause much relief or actual cure can be afforded by appropriate treatment. Many cases are overlooked, some. times perhaps to the advantage of the patient, either because the practitioner does not search for them or because he does not possess the necessary taotus eruditus. There have, unfortunately, been false prophets in this as in other departments of medicine. There has been a tendency of late years to make an unwarrantable use of the fact that a patient bas a moveable kidney as a convenient peg on which to hang any combination of symptoms for which no obvious explanation can be found. On this subject it is necessary to speak out with no uncertain sound. It is important to know that the kidneys are often abnormally moveable without giving rise to any inconvenience whatsoever and that this mobility may be associated with symptoms of dyspepsia, neurasthenia, or even insanity without being the fons $t$ t origo of these maladies. I strongly deprecate such teaching as that "minor degrees of mobility of the kidneys are the unreco. gnised cause of much suffering and distress." I do not think there is a particle of evidence that this is the case and I regard the slight degrees as of trivial importance. The majority of cases of even freely moveable kidney are unattended with symptoms and require no treatment directed to that condition.

I would first then direct your attention to the commonness of moveable kidneys. Many of us can remember the time when the condition was looked upon as unusual and specially interesting on account of its rarity. Some, indeed, such as Lawson Tait, a quarter of a century ago denied, for a time, its existence altogether. I saw but few cases when I was a student and comparatively few until I started on the investigation which has led to this paper. The reasons why the condition was thought to be rare were that in the absence of local symptoms it was not looked for and that often the examination was not conducted in the proper way. I may quote in support of this view a statement from a paper published in 1897 by an American authority, surgeon-in-chief to a large hospital for women in an important American city. He says: "For some years, in the more or less systematic examination of office patients, I looked in vain for a case of moveable kidney. After 1893 I began systematically to look for moveable kidneys in all cases having the rational symptoms of this disorder. These examinations were all made with the patient lying on the back upon the office table and were entirely fruitless." This is a candid and instructive confession and all honour to the surgeon for recording the fact that he did not know at that time how to conduct the examination. It is possible that some of us still miss moveable kidneys through not examining in the proper way, and therefore at the risk of repeating what is probably familiar to most of my hearers 
to-night I shall state briefly the method of examination which I bave adopted in these investigations.

The patient lies on the couch perfectly flat on the back, the head as low as the shoulders, and the legs extended. The examiner sits on the right of the patient when examining the right side, and on the left when examining the left side. The examiner first gently palpates the right side of the abdomen with the right hand from below upwards. If he finds all normal he then places the left hand behind under the back below the last rib and makes firm pressure upwards. The right hand is then placed flat on the abdomen in front and palpation is made between the two hands. Often the kidney can then be felt between them. The patient is at this stage asked to take a series of long deep breaths and the lower end of the kidney will then likely be felt to descend with inspiration. In cases where there is abnormal mobility you feel the kidney descend so far that you can slip the upper border of the right hand above the upper end of the kidney. By putting forward the thumb of the left hand above the kidney you can then fix it in its descended position and release the right hand, which can be employed to palpate the whole of the organ and determine the extreme limits of its mobility. When you remove the thumb of the left hand the kidney can be felt to slip back into its position. A similar investigation is made on the left side, the hands being reversed, the right being underneath and the left above.

This method is perfectly simple, and I believe one seldom fails by its means to discover the degree of mobility of the kidneys. It is only by practice that one become an adept at it, and I find students seldom succeed at first in making out the kidney mobility. But the method is most valuable, not only for making out the mobility of the kidneys but also for investigating other abnormal conditions of the two sides of the abdomen. Some have advocated that the examination should be conducted with the patient standing up, but I am sure that position has no advantages, and has very obvious disadvantages as a routine method, although sometimes it is useful to employ it in addition to the other.

In the record of my examinations I have defined what I call a moveable kidney as one in which I have been able to get completely above the upper end of the kidney. Those cases in which I have been able to feel a considerable part of the kidney but have not succeeded in getting above it, I have called palpable kidneys. I have not thought it worth while recording those cases in which the lower end of the kidney only could be felt-they are so numerous. A certain degree of mobility is perfectly normal. To call a kidney of which one can merely feel the lower end below the ribs a "dropped" or "dislocated" kidney is a "terminological inexactitude."

With this preliminary clearing of the ground I shall now proceed to give you some of the results of my examinations. First, 2801 females of all ages were examined. Among these there were 449 cases in which the kidneys were palpable, as I have defined it, and 515 in which the kidneys were moveable. That is to say, that in 16 per cent. the kidneys were palpable only, and in an additional 18.4 per cent. they were not only palpable but freely moveable. Secondly, 1067 males of all ages were examined. Among these were 25 cases in which the kidneys were palpable and 11 cases in which they were moveable, or 2.3 per cent. of palpable kidneys and 1 per cent. of moveable. I have made out tables, which I shall append, giving the exact numbers examined at different ages, with the results, of which I shall here give a short summary, with some details which are of interest.

Under the age of 12 years I met with no case in which the kidneys were moveable. 99 female children under 12 years of age were examined. In ten the kidneys were palpable, but none came into the moveable group. Between the ages of 13 and 16, 136 females were examined, among whom 19 cases of palpable kidneys were found and seven moveable. The earliest age at which $I$ found a moveable kidney was 13 years. 293 females between 17 and 19 years of age were examined, among whom were 42 cases of palpable and 35 cases of moveable kidneys. Thus of quite roung women 12 per cent. had moveable kidneys. Between 20 and 22 years of age about the same proportions were met with. Among 333 females between these ages there were 49 cases of palpable and 42 cases of moveable kidneys, or $12 \cdot 6$ per cent. When we get over the age of 22 years there is a remarkable increase in the proportion of cases. Of females between the ages of 23 and 29 years 647 were examined, among whom were 120 cases of palpable and 139 cases of moveable kidneys, so that the proportion of moveable lidneys has risen to $21 \cdot 4$ per cent. Between the ages of 30 and 37 years 612 females were examined, among whom were 109 cases of palpable and 165 cases of moveable kidneys, a proportion of nearly 27 per cent. - that is to say, that between these ages one out of every three or four women examined had moveable kidneys. After the age of 37 years the proportion declines again. 569 women between 38 and 54 years of age were examined among whom were 90 cases of palpable and 111 cases of moveable kidneys, a proportion of just about 20 per cent. Between the ages of 55 and 86 years 112 females were examined, with 10 cases of palpable and 16 of moveable kidneys, the proportion thus having fallen to 14 per cent.

Among the males no case of moveable kidney was met with under 20 years of age. The highest proportion was between the ages of 30 and 40 years, five cases occurring among 282 men, or nearly 2 per cent.

The only series of cases known to me which can challenge comparison with my own is that of Glénard, who examined 4215 patients and found among them 537 cases of moveable kidney. There were 485 cases among 2202 women and 52 cases among 2013 men-that is, 22 per cent. among the women, and 2.7 per cent. for the men. These figures are in striking agreement with my own. Lindner's statement that one out of every five or six women has a moveable kidney is strictly correct if we modify it as follows :-One out of every five female patients has a moveable kidney. Lindner adds with perfect truth that it is the most frequent anomaly in the female subject. Such statistics as those of Senator and Skórczewsky, quoted by Professor A. Macalister in his article in Allbutt's System, must have been founded on very imperfect examinations. The former states that moveable kidney is as common among the rich as among the poor and but one case is met with among 139 sick women. The latter gives the proportion as 3.1 per cent. among females and 0.76 per cent. among males. On the other hand, an observer who makes the astonishing statement that of 100 women and 100 men whom he had seen during the past few weeks in private 42 women and six men had dropped kidney may be said to have a somewhat exaggerated idea of what constitutes that condition.

With regard to moveable kidney in young children, the number of cases I have examined may, perhaps, seem small. Still, I think the conclusion is warranted that in children under 12 years of age moveable kidney is rare. Legendre carefully examined a large number of children, and only found one case in a girl aged 14 years, who had mobility of the left kidney of the first degree. Cases in young children have been recorded, however, by good observers. Of the 526 cases of moveable kidney, there was only one case where the mobility was only on the left side. In 49 cases the kidneys were moveable on both sides. In 476 cases the mobility was abnormal only on the right side. The patients examined were suffering from a variety of complaints, and it is instructive to note the proportions in the different maladies. There were 138 cases of morbus cordis, and of these there were 34 cases of moveable kidney, or $24 \cdot 6$ per cent., which, curiously, is the highest percentage. There were 602 cases of dyspepsia with 143 cases of moveable kidney, or 23.7 per cent. There were 463 cases of pulmonary tuberculosis with 101 cases of moveable kidney, or $21 \cdot 8$ per cent. There were 562 cases of bronchial catarrh with 91 cases of moveable kidney, or $16 \cdot 2$ per cent. There were 354 miscellaneous cases with 83 cases of moveable kidney, or 23.4 per cent. There were 128 cases of anæmia with 11 cases of moveable kidney, or 8.6 per cent. It appears, therefore, that those suffering from chest complaints contributed even more than their due share of cases of moveable kidney, and there is no ground for supposing that those patients who suffered from dyspepsia owed their digestive trouble to the abnormal condition of their kidneys.

It has long been supposed that child-bearing has an important influence in the production of abnormal kidney mobility, but my investigations do not lend much support to this supposition. Age, I think, is a much more important factor. 211 women from 18 zears of age upwards who had borne one child were examined. Of these 50 had moveable kidneys, or 23.7 per cent. 199 women from 19 years of age upwards who had borne two children were examined. Of these 63 had moveable kidneys, or $31 \cdot 6$ per cent. This, it is true, is a very high proportion, but the proportion becomes smaller again with a larger number of pregnancies. Thus 173 women from 21 years of age upwards who had borne three children contributed 52 cases of moveable kidney, or 
30 per cent. Among 133 women from 24 years upwards who had borne four children, there were 24 cases, or only 18 per cent. ; among 93 women from 26 years upwards who had had hive children there were 21 cases, or $22 \cdot 5$ per cent. among 158 women from 27 years upwards who had had six or seven children there were only 22 cases, or 13.9 per cent. and among 174 women from 34 years upwards who had had eight or more children there were 29 cases, or 16.6 per cent. On the whole, therefore, the influence of childbearing on the production of moveable kidney is not very great. This conclusion agrees with the statistics of Lindner and the experience of Edebohls, Kuttner, and Walch.

Among the causes of abnormal kidney mobility the influence of corsets, tight-lacing, and dress has been considered as important. I do not think, however, that the class from which my patients have been drawn can be accused of tight-lacing to any great extent. Edebohls maintains that tight-lacing is the most effective agent which can be applied to maintain a kidney in its normal position. Laxity of the abdominal walls cannot play a very important rôle, for its most common cause ; repeated child-bearing, as we have seen, has no decided influence. Probably absorption or atrophy of the perirenal fat has an important influence, and certainly the condition is more often met with in wasted individuals. Sex, however, is in some way the most important factor in causation, and B scquet's suggestion that an increase of volume of the kidney at the catamenial period plays a part in cansation may have some truth in it. The mobility may be produced suuddenly as the result of strain or traumatism, of which clinically one occasionally meets with undoubted instances. In those rare cases where there is a meso-nephron the abnormal mobility is obviously a congenital condition. The reason why the right kidney is so much more frequently moveable than the left is no doubt anatomical. The liver tends to displace the right the pancreas to support the left. The rarity of moveable kidney in children is probably due to the fact that the tissue of the capsule of the kidney until the tenth year is simply areolar and does not become filled with fat until after that age.

Symptoms.-Out of 515 cases with the high degree of mobility which I have defined, no fewer than 411 had no symptoms which with an reasonableness could be attributed to the condition of the kidneys. This supports what Professor Osler bas said, that in a majority of the cases there are no symptoms whatever. One might truly say in a vast majority. Now what is the right course to adopt when we find a freely moveable kidney which produces no trouble of any kind? I think it is best that wa should tell the patient that the kidney is moveable but explain that it is a common condition among women, that it is giving no trouble, may possibly never give any trouble, and at the present time requires no treatment. There is always the risk of such a patient falling into the hands of somebody who will want to treat the condition, in spite of the absence of symptoms, and the patient will think you have missed something of importance. One of my hospital patients having heard me say that she had a moveable kidney went to another hospital and was operated on by an eminent surgeon. She came back afterwards to tell me she was no better for the operation As she had bad no symptoms referable to the kidney I did not expect she would be. While a moveable kidney may have given no trouble up to the time it is discovered by the physician there is always the possibility of trouble arising in the future. A caution should always be given against lifting heavy weights or straining the abdomen. I do not consider $i t$ is necessary to put restrictions on ordinary modes of exercise, but I think cycling and working a foot sewing machine are likely to do harm.

I saw recently a woman, aged 38 years, who during the last year had had symptoms arising from a freely moveable right kidney. She told me that when she was 18 years of age she had been informed that she had a "floating kidney" Nothing had been done for it and she had thus remained free from symptoms for over 18 years.

In 104 cases the mobility of the kidneys was the canse of symptoms varying in degree. By far the most constant symptom, the symptom almost invariably present if symptoms were present at all, was pain. The pain was usually referred to the right lumbar region behind but sometimes more to the middle or all round the back, sometimes to the right hip or thigh or down the leg, sometimes in front to the right iliac fossa, sometimes to the epigastrium, sometimes to the ovarian ragion or to the groin. In 15 cases the pain was only felt in the left lumbar region. In seven of these cases both kidneys were moveable but in the other eight the right kidney alone was freely moveable. In some other instances where both kidneys were moveable pain was felt on both sides but was worse on the left. The pain was usually of a dragging or dull aching or bearing-down character, but some. times more severe stabbing or sharp knife-like pains were experienced. Patients have described the pain to me as so severe as to double them up. The pain is, as a rule, not constant, but comes and goes. The pain is made worse or may only be exporienced after walking or standing, or working a sewing-machine, or after a hard day's work. The pain may be induced or aggravated by coughing. It is usually much relieved or disappears altogether on lying down, but sometimes posture fails to give ease and on account of the pain the patient may lie awake all night. Usually the most comfortable position is on the right side with the legs drawn up or lying on the back. The pain is sometimes aggravated at the menstrual periods.

The following are characteristic accounts obtained from patients : "The pain in the right flank has occurred when the patient has had much standing and has generally been absent on Saturday and Sunday when she is not at work The pain is of a jumping character as if something were moving about inside." "There has been a dragging pain in the right side for about a year. It is worse after exercise and sometimes is attended with a feeling of sickness. Some times the pain lasts for days. The onset of the pain is related to what the patient has been doing. Working a hand sewing machine always makes it worse. Walking, scrubbing \&c., also produces this effect. Rest always relieves it. Great relief was obtained from a pad and bandage."

Edebohls has described as a characteristic symptom epigastric pain, independent of food, situated at a point five to six centimetres to the left of the median line at or nea the free border of the left costal cartilage, but I have met with no examples of this. Very few of the patients included in this investigation were conscious of the presence of any tumour. One patient, in whom the kidney could be displaced to the right iliac fossa, had discovered that a lump of the size of a hen's egg made its appearance above the groin when she had an attack of pain. The attack of pain was accompanied by retching and sickness and the passage of a large quantity of urine. The attack would last about eight hours and when it passed off the lump would disappear with a click. Another patient said that she was conscious of the presence of a lump in the right kidney region on coughing and straining; another that a lump came above the right groin when she lifted anything. Two other patients said that they were conscious of the presence of a lump in the right side of the abdomen. Another patient, a woman, aged 4 years, whose left kidney alone was moveable, complained o pain and swelling on the left side of the "stomach," the swelling having gradually become large. Another patient, who had a freely moveable right kidney, suffered trom attacks of pain in the right lower abdomen and said that she had the sensation of a lump resting on the hip bone when the attack was on. In six cases freely moveable kidneys have been associated with an irritable condition of the bladder and a frequent and irresistible desire to micturate.

As long as we are dealing with local symptoms we are on sure ground. The symptoms are unmistakable. They disappear, as a rule, with rest in the proper position and can bo cured or alleviated by suitable support to the erring organ. But when we come to the long list of symptoms with the production of which moveable kidneys have been credited we must preserve a judicial mind and sift the evidence such as it is. A large number of the patients who consult us suffer from dyspepsia, but the proportion of dyspepti s who have moveable kidneys is no larger than that of patients sufferin from pulmonary tuberculosis or morbus cordis. The connexion of dyspepsia with moveable kidneys is not, therefore. one of effect and cause. In a certain number of cares we find th abnormal mobility of the kidneys associated with dropping o other abdominal organs, and the dropuing of the stomach i connected with troublesome dyspepsia, but the treatment of the kidney condition alone will not cure that. In a good many in-tances, however, we learnt that the patient bad suffered from attacks of vomiting which were clearly the result of the displacement of the kidneys. The e attacks were accompanied with a feeling of sickening pain in the renal region and the vomiting passed off when the pain did. These were examples of Dietl's crises. When we have an opportunity of examining the patient during an attack we find, in addition to the sab. jective pain, swelling and tenderness of the affected kidney. 
One patient, a woman, aged 22 years, suffered from very marked symptoms of this kind. At the age of 19 years she was in bed on and off for ten months with pain more or less generally diffused over the abdomen, but worse in the region of the left kidney, and with frequent attacks of sickness. She bad a second similar attack lasting two or three months two years later. Both kidneys were very freely moveable.

All kinds of nervous symptoms have been attributed to moveable kidneys. Even locomotor ataxy has been thought to have a causal connexion, because someone found six cases of moveable kidney among 24 women suffering from locomotor ataxy, which is just the proper proportion. Migraine, vertigo, all kinds of neuralgias, epilepsy, neurasthenia, hypo chondriasis, and various forms of insanity have been at divers times referred to moveable kidneys as their cause. Search among these cases and you will find the same proportion of moveable kidneys as in other complaints, but there is not a tittle of evidence that there is any causal relation between them. In an unusually large proportion of cases of neurastlenia moveable kidneys are no doubt to be found, but there were not more than about ten of such cases in the present series. Osler kas truly said that "in a thin person, male or female, who presents the general symptoms of neurasthenia, you will be almost certain to find, on examination, mobility of one or other or of both of the kidneys." The patient who comes with the "petits papiers" of Charcot is almost certain to have moveable kidney. The knowledge that they have moveable kidneys is to some patients a source of mental distress.

Dislocation of the kidney may take place more or less suddenly. A typical history is as follows. While lifting a heavy table a girl, aged 17 years, was seized with severe pain in the right loin, worse at the back but passing down round to the iliac region. The attacks of pain lasted about 24 hours. Poultices, \&c., were applied to relieve the pain. Since that time similar attacks occurred at intervals, one lasting as long as three days. The right kidney was freely moveable. Another patient, a girl, aged 18 years, who had had aching pain in the right loin for about three months, was seized with acute pain in the same situation while lifting furniture, the pain being so severe that she fell down. In another case, a girl, aged 20 years, while standing at work was suddenly seized with such acute pain in the right loin that she nearly fell to the ground and became covered with a cold, clammy sweat. From that time she continued to suffer more or less from pain in the right loin. Another patient, a woman, aged 30 years, attributed her trouble to lifting her husband out of bed. Pain in the right side came on then and persisted from that time. Her right kidney was found to be very freely moveable.

$I$ append here in tabular form details of the female and male cases examined, giving the numbers of the former examined at different ages and the proportion of palpable and moveable kidneys found, as well as the numbers in which symptoms were present as the result of the kidney condition.

Treatment. - The vast majority of the cases had, as I have pointed out, no symptoms connected with the kidney condition and required no treatment for it. I have already indicated the nature of the advice which I consider should be given to such patients. Cases in which the patient suffers from attacks of pain of only occasional occurrence are best treated by rest in the position which the patient instinctively assumes as the most comfortable-namely, on the back or on the side of the affected organ. The local application of heat by hot-water bottle or hot fomentations, or of preparations of belladonna are useful in relieving severe pain. In cases where the pain is wearing and persistent, rest in bed should be insisted on for some time. In thin and neurasthenic subjects an attempt should be made to improve their nutrition and general condition. Prolonged rest, good feeding, and general massage sometimes prove very beneficial. The kidney may settle down into its proper situation, and with an increase of the body fat be better supported when the patient gets about again. A pad and ka idage will often afford great relief when the patient is up and about, and in the slighter cases the patient need only wear it when she is about to make some unusual exerticn. Many of the kidney belts ar. cumbrous and unsatisfactory and fail to give relief. The appliance which $I$ have found most efficient is of the nature of a spring truss, and keeps the kidney in position by properly applied pressure of the spring. Some patients are entirely relieved from their symptoms by such an apparatns

It is interesting to note that of the patients who rad eymptoms there was not one in this series in whom the
Table of Female Cases.

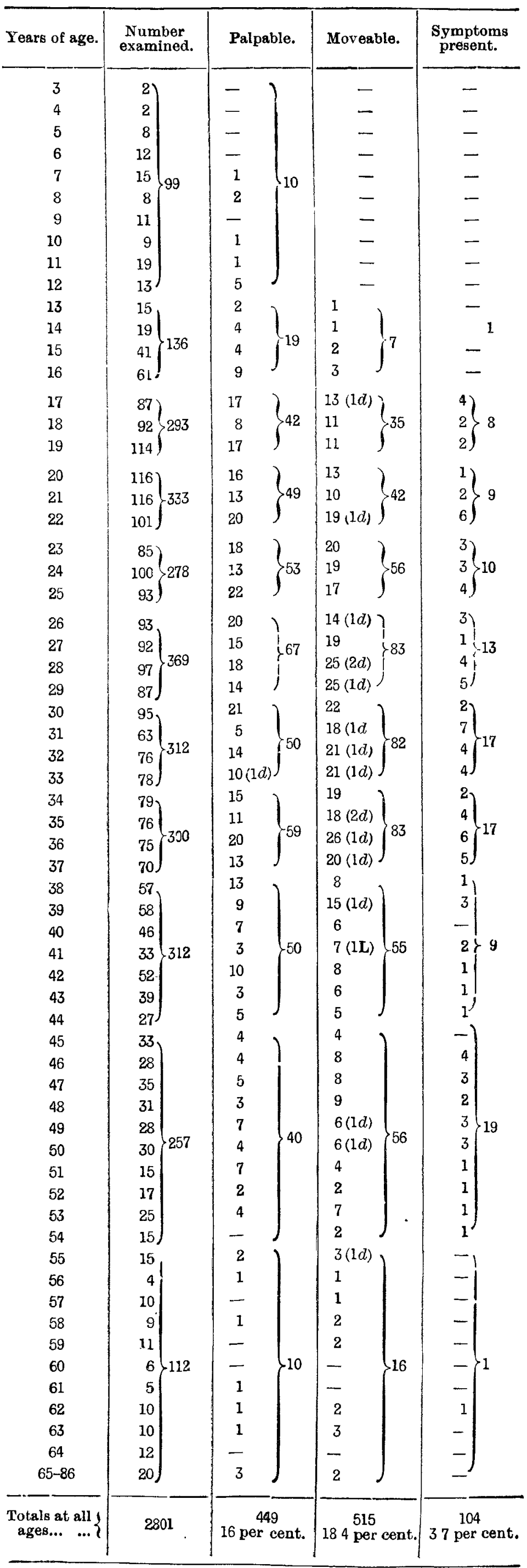


1144 The Lancet,] DR. B. DAWSON : ROENTGEN RAYS AND STRICTURE OF (ESOPHaGUS. [OCt. $26,1907$.

\begin{tabular}{c|c|c|c|}
\hline \multicolumn{3}{|c|}{ Table of Male Cases. } \\
\hline Years of age. & $\begin{array}{c}\text { Number } \\
\text { examined. }\end{array}$ & $\begin{array}{c}\text { Number } \\
\text { palpable. }\end{array}$ & $\begin{array}{c}\text { Number } \\
\text { moveable. }\end{array}$ \\
\hline Under 5 & 1 & - & - \\
10 & 17 & - & - \\
15 & 33 & - & - \\
20 & 127 & 2 & - \\
25 & 166 & 3 & 1 \\
30 & 182 & 6 & 2 \\
35 & 166 & 1 & 2 \\
40 & 116 & 4 & 1 \\
45 & 89 & 3 & - \\
50 & 65 & 3 & - \\
60 & 84 & & - \\
Over 60 & 21 & & - \\
\hline
\end{tabular}

Total 1067 cases. 25 palpable; 2.3 per cent. 11 moveable ; 1 per cent. symptoms were so severe or serions as to call for surgical treatment. I can recollect very few cases altogether in which I have advised patients to undergo an operation. I am inclined to think that most of the cases which surgeons operate on go to them directly and not through the advice of a physician. When I have a case where the symptoms are severe and persistent and do not yield to medical treatment, I am glad to seek the help of the surgeon. But I should always advise recourse to other methods of treatment first. While I have no doubt that the results of surgical fixation of the kidney are sometimes mosts excellent, the operation is not uniformly successful, and is not altogether free from risk to life. Just the other day I got a letter from a friend in the country which contained the following brief report : "I have been so much occupied this last week, as I have had a poor girl staying with us whose mother died in the nursing home at ...... She underwent an operation for floating kidney and died a fortnight after. It is really very $\$$. $A$, as she leaves a large family."

I must leave it to surgeons to describe the methods and indications for operation. I may, however, give a short summary of the results in 131 cases which $I$ have collected from literature. Out of 131 recorded cases operated on by eminent surgeons, nephrorrhaphy or nephropexy was performed 113 times, with a successful issue in 80 cases, but in some of these the time which had elapsed after the operation was too short to enable one to judge of the real result. In 17 cases partial relief only followed, and in 13 the result was failure, and in five of these subsequent nephrectomy was performed with a successful issue. In three cases there was a fatal result. Nephrectomy was performed as the primary operation in 18 cases : 14 were successful ; one was a failure; three proved fatal. In these cases which were operated on pain was an almost invariable symptom. Dietl's crises were recorded in two cases; vomiting was recorded in 11 ; irregular micturition in one; the presence of a tumour was noted in 10 ; and mental distress on account of the tumour in one. There is one surgeon who seems to have operated in a large proportion of his recorded cases on account of such symptoms as nervousness and dyspepsia.

As it is now eight years since I collected from what was then recent literature the above series of cases operated on, I have consulted the surgical reports of St. Thomas's Hospital for the six years ending December, 1905, and I wish to add that the results of operation appear to be much better in recent years than they were previously. Of 106 cases of nephroptosis admitted to the surgical wards of St. Thomas's Hospital during these years, 80 cases were operated on without a single death. In 73 cases the operation was nephro. pexy, in three nephropexy was combined with nephrotomy, in one the operation was a lumbar incision and packing with gauze, in one nephropexy was followed by subsequent nephrectomy, and in two nephrotomy alone was performed.

In conclusion, I may repeat that moveable kidney is undoubtedly a very common condition in the female subject, that in the great majority of cases it produces no symptoms and that it requires no treatment when local symptoms are absent. When symptoms are present a fair trial should be made of non-operative methods of treatment. Only when such methods have failed to relieve, or when there is reason to believe that the kidney is the seat of disease, should recourse be had to operation.

Upper Brook-street, W.

\section{ROENTGEN RAYS AS AN AID TO THE DIAGNOSIS OF STRICTURE OF THE CESOPHAGUS.}

\section{BY BERTRAND DAWSON, M.D., B.Sc. LOND.,} F.R.C.P. LOND.,

PHYSICIAN EXTRAORDINARY TO HIS MAJESTY THE KING; PHYSICLAN AND PATHOLOGIST TO THE LONDON HOSPITAL.

THE method adopted is as follows. The observer stands inside a dark sentinel-box for ten minutes before the observation is made: this is essential if his vision is to be at its best; the screen, on a moveable frame, is let into one side of the sentinel-box; the patient is stationed in front of it, either with the anterior surface of his chest towards the screen or, which often gives better results, with his right lateral chest against it and the right arm uplifted. When all is ready the patient takes a mouthful of thick emulsion of bismuth and when the word is given by the observer swallows it.

The illustrations accurately represent two cases. They were sketched in the rough on a piece of transparent paper fixed against the screen. artially finished drawings were then made and were corrected by subsequent observations.

CASE 1.-In Case 1 as each mouthful of bismuth was swallowed a definite black mass appeared at $\Delta$ and moved downwards, manifesting as it did so a fainter, though still definite, shadow, as shown in Fig. 1. At B a black pouch appeared which became darker and bigger as subsequent monthfuls were swallowed. When this pouch, $\mathrm{B}$, became sufficiently distended, suddenly one saw a black stream, $\mathrm{C} \mathrm{D}$ shoot forth from the centre of the lower end and make its way quickly downwards through the diaphragm E. This stream shot out like a rocket and for some reason was interrupted as shown in the illustration. The observation was made with the patient's right lateral chest against the screen, with the right arm upraised. F represents the faint shadow of the heart. The stricture was evidently a considerable one opposite the fifth dorsal vertebra and the cesophagus was dilated above it. The illustration explains why a bougie would sometimes get through the stricture and at other times not, for it would only get through if it happened to hit the aperture in the centre of the sac.

The examination by means of the screen was in this case particularly helpful from the fact that early in the illness the patient brought up, after a bougie had been gently and cautiously passed, a large quantity of blood. The patient stated the quantity of blood to be four quarts (which was probably an exaggeration), and according to his description it was regurgitated rather than vomited. The possibility, therefore, of the difficulty of swallowing being due to aneurysm was a real one, otherwise there was nothing peculiar about the history. The patient was 54 years of age; he ha $\hat{\alpha}$ increasing difficulty of swallowing, with slight pain referred to the inferior angle of the right scapula, regurgitation of food, and wasting. Bougies stopped 13 inches from the teeth. He died from septic pneumonia, his illness having lasted about a year. The necropsy confirmed the diagnosis of carcinoma of the cesophagus, the septic pneumonia having been caused by ulceration into the left bronchus.

CASE 2.-The observation was made with the left side of the patient against the screen. In this case the bismuth as it was swallowed mapped out the track A B and was evidently arrested at the level of the diaphragm $E$. There was some dilatation at $B$ but considerably less than in the preceding case. A faint shadow of the heart was seen at $C$. The illustration (Fig. 2) discloses a further matter of interest: the light area $f$ beneath the diaphragm $\mathbf{s}$ is the upper portion of the stomach filled with air, and the level surface, $\mathrm{H}$, limiting this light area below was fluid, occupying the lower portion of the stomach, and when the patient was shaken waves could be distinctly seen on the surface of the fluid. Incidentally it may be remarked that a very efficient way of mapping out the position of the stomach is to inflate it with gas in front of a screen. I have frequently adopted this method and have found it useful.

The clinical features of this case pointed to an obstruction of the osophagus at its extreme lower end. A No. 23 bougie was stopped at 17 inches from the teeth; smaller bougies were similarly stopped, but No. 11, having first encountered resistance, was felt to ride over something hard and to go 